\title{
The association of follicular fluid volume with human oolemma stretchability during intracytoplasmic sperm injection
}

\author{
Taketo Inoue ${ }^{1,2}$, Yoshiki Yamashita' ${ }^{1}$, Yoshiko Tsujimoto' ', Shuji Yamamoto', Sayumi Taguchi' ${ }^{1}$, Kayoko Hirao $^{1}$, Mikiko Uemura $^{3}$, \\ Kayoe Ikawa', Kazunori Miyazaki ${ }^{1}$ \\ ${ }^{1}$ Umeda Fertility Clinic, Osaka; ${ }^{2}$ Department of Emergency, Disaster and Critical Care Medicine, Hyogo College of Medicine, Nishinomiya; ${ }^{3}$ Faculty of \\ Rehabilitation, Kobe Gakuin University, Kobe, Japan
}

Objective: Oocyte degeneration often occurs after intracytoplasmic sperm injection (ICSI), and the risk factor is low-quality oocytes. The follicular fluid (FF) provides a crucial microenvironment for oocyte development. We investigated the relationships between the FF volume aspirated from individual follicles and oocyte retrieval, oocyte maturity, oolemma stretchability, fertilization, and development.

Methods: This retrospective study included data obtained from 229 ICSI cycles. Ovarian stimulation was performed according to a gonadotropin-releasing hormone antagonist protocol. Each follicle was individually aspirated and divided into six groups according to FF volume $(<1.0$, 1.0 to $<2.0,2.0$ to $<3.0,3.0$ to $<4.0,4.0$ to $<5.0$, and $\geq 5.0 \mathrm{~mL}$ ). Oolemma stretchability during ICSI was evaluated using a mechanical stimulus for oolemma penetration, that is, the stretchability was assessed by oolemma penetration with aspiration (high stretchability) or without aspiration (low stretchability).

Results: Oocyte retrieval rates were significantly lower in the $<1.0 \mathrm{~mL}$ group than in the $\geq 1.0 \mathrm{~mL}$ groups $(46.0 \%$ [86/187] vs. $67.5 \%-74.3 \%$ [172/255 to $124 / 167]$, respectively; $p<0.01$ ). Low oolemma stretchability was significantly more common in the $<1.0 \mathrm{~mL}$ group than in the $\geq 1.0 \mathrm{~mL}$ groups during ICSI (22.0\% [13/59] vs. 5.8\%-9.4\% [6/104 to 13/139], respectively; $p=0.018$ ). There was a relationship between FF volume and oolemma stretchability. However, there were no significant differences in the rates of fertilization, cleavage, $\geq 7$ cells at day 3 , and blastocyst development among all groups.

Conclusion: FF volume is potentially associated with the stretchability of metaphase II oolemma during ICSI. Regarding oolemma stretchability, ensuring a uniform follicular size during ovarian stimulation is crucial to obtain good-quality oocytes.

Keywords: Fertilization; Gonadotropin-releasing hormone; Infertility; Membranes; Oocytes; Ovarian follicle; Ovulation induction; Sperm injections, Intracytoplasmic

\section{Introduction}

During intracytoplasmic sperm injection (ICSI), the human oolemma may be penetrated without additional mechanical stimuli, such as aspiration and piezo-pulses, several times because of low oolem-

Received: Apr 17, 2017· Revised: Jul 21, 2017· Accepted: Aug 17, 2017 Corresponding author: Taketo Inoue

Umeda Fertility Clinic, 3-17-6, Toyosaki, Kita-ku, Osaka 531-0072, Japan

Tel:+81-6-6371-0363 Fax:+81-6-6372-0585 E-mail: taketoein@yahoo.co.jp

This is an Open Access article distributed under the terms of the Creative Commons Attribution Non-Commercial License (http://creativecommons.org/licenses/by-nc/4.0/) which permits unrestricted non-commercial use, distribution, and reproduction in any medium, provided the original work is properly cited. ma stretchability/flexibility. Subsequently, oocyte degeneration is occasionally experienced. The incidence of oocyte degeneration after ICSI has been reported to be $5 \%$ to $15 \%[1,2]$, and the risk factors involve oocyte quality [3-7] and operator skill/technique or use of microtools [8-10]. Rosen et al. [11] reported that oocyte degeneration following ICSI is not technician-dependent and that degeneration is likely related to inherent oocyte quality in patients who are under ovarian stimulation.

Previous studies have reported on the relationship between human oocyte degeneration following ICSI and oocyte cytoplasmic viscosity $[3,4]$, oolemma stretchability/flexibility $[5,6]$, or oolemma fra- 
gility [7]. Krause et al. [3] investigated the formation and persistence of the obligatory injection funnel after conventional ICSI and found that the funnel volume was higher in normal fertilized oocytes than in degenerated oocytes after conventional ICSI. Iwayama et al. [5] reported that the survival rate following piezo-ICSI was lower with low oolemma stretchability than with high oolemma stretchability. Moreover, Iwayama et al. [5] showed that the implantation rate was lower following low-stretchability oocyte-derived embryo transfer than following high-stretchability oocyte-derived embryo transfer. Therefore, for oocyte survival after ICSI, oocyte quality is crucial.

The follicular fluid (FF) provides a crucial microenvironment for follicular development, with chemical components such as hormones, growth factors within the transforming growth factor- $\beta$ superfamily, interleukins, and antiapoptotic factors $[12,13]$. The size of the follicle that provides the microenvironment to the oocyte has been shown to be related to steroid hormone levels in the follicle [14], and steroid hormone levels in an individual follicle have been reported to affect oocyte maturity [15] and fertilization outcomes after ICSI [15,16]. Moreover, follicle size/volume is related to oocyte maturity $[17,18]$, and a cumulus-oocyte complex (COC) derived from $<1.0 \mathrm{~mL}$ of FF has been shown to be associated with a low in vitro fertilization rate [19]. Therefore, follicle size/volume is associated with oocyte quality. Several previous studies have analyzed the relationship between fertilization outcome and oolemma/cytoplasm quality [3-7], as quality is a key factor for survival following ICSI; however, few reports have discussed the relationship between FF volume and oolemma/cytoplasm quality. The goal of the present study was to investigate the relationship between the FF volume aspirated from individual follicles and rates of oocyte retrieval, oocyte maturity, oolemma stretchability, fertilization, and development during ICSI.

\section{Methods}

\section{Patients}

This retrospective study was conducted using data from 229 ICSI cycles performed between February and September 2015. The mean ( \pm standard deviation) age of the women was $39.0 \pm 4.7$ years (range, 26-47 years). Informed consent was obtained for all procedures. All procedures were performed in accordance with the Declaration of Helsinki.

\section{Ovarian stimulation/oocyte retrieval}

Ovarian stimulation was performed according to a gonadotropin-releasing hormone (GnRH) antagonist protocol. A GnRH antagonist (Cetrotide; Shionogi \& Co., Osaka, Japan) and human menopausal gonadotropin (ASKA Pharmaceutical Co., Tokyo, Japan) were used in the protocol. When at least two follicles reached $18-20 \mathrm{~mm}$ in diameter (as determined by transvaginal ultrasonography), 5,000 IU of human chorionic gonadotropin (Fuji Pharma Co., Tokyo, Japan) was administered. Oocyte retrieval was performed 36 hours after the human chorionic gonadotropin injection. The diameter of each follicle was measured, and each follicle was individually aspirated and collected. The FF volume aspirated from each follicle was measured using a 10-mL syringe (Terumo Corp., Tokyo, Japan) that was connected to an ovum pick-up needle (Kitazato OPU Needle; Kitazato Corp., Shizuoka, Japan) on first aspiration. The FF then was decanted into a 60-mm dish (Corning, Corning, NY, USA) and the COC was harvested. If no COC was obtained in the original aspiration, flushing was performed five times. Initial aspirates, which contained large amounts of blood, were excluded from the analysis. FF volume was divided into the following six groups: group $\mathrm{A}$, $<1.0 \mathrm{~mL}$; group $\mathrm{B}, 1.0$ to $<2.0 \mathrm{~mL}$; group C, 2.0 to $<3.0 \mathrm{~mL}$; group $D, 3.0$ to $<4.0 \mathrm{~mL}$; group $\mathrm{E}_{1} 4.0$ to $<5.0 \mathrm{~mL}$; and group $\mathrm{F}, \geq 5.0$ $\mathrm{mL}$. Each $\mathrm{COC}$ was individually obtained, placed into a 5-mL Falcon round-bottom tube (Corning) containing HFF99 medium (Fuso Pharmaceutical Industries, Osaka, Japan) with a 10\% serum protein substitute (Kitazato Corp.), and cultured for 2 to 3 hours until denudation.

\section{Intracytoplasmic sperm injection}

Oocytes were freed from cumulus cells using $0.05 \%$ hyaluronidase (Sigma-Aldrich, St. Louis, MO, USA) dissolved in modified human tubal fluid medium (Kitazato Corp.) by pipetting. After denudation, metaphase II (MII) oocytes were cultured for $\geq 1$ hour [20]. Immobilization of motile sperms was performed in a $5-\mu \mathrm{L}$ drop of $5 \%$ polyvinylpyrrolidone (Irvine Scientific, Santa Ana, CA, USA) dissolved in a modified human tubal fluid medium with a $10 \%$ serum protein substitute. Oocytes were inseminated through ICSI using injection pipettes (inner diameter, $4.7 \mu \mathrm{m}$; outer diameter, $6.0 \mu \mathrm{m}$ [Cook Medical, Bloomington, IN, USA]) [21]. Oolemma stretchability during ICSI was evaluated using a mechanical stimulus for oolemma penetration, that is, the stretchability was assessed by oolemma penetration with aspiration (high stretchability) or without aspiration (low stretchability). On the following day, oocyte fertilization and degeneration were confirmed. Fertilized eggs were individually cultured (in 6\% $\mathrm{CO}_{2}, 5 \% \mathrm{O}_{2}$, and at $37^{\circ} \mathrm{C}$ ) in a $20-\mu \mathrm{L}$ drop of Global total medium (LifeGlobal Group, Guilford, CT, USA) covered with light mineral oil (Oil for Embryo Culture, Irvine Scientific) on a $60-\mathrm{mm}$ dish until day 6 , until the embryo was cryopreserved at day 3 , or until a fresh embryo transfer was performed. The medium change was performed at days 1, 3, and 5.

\section{Statistical analysis}

The mean age of the patients was evaluated using one-way analysis of variance. Consequently, the mean age of the patients was evaluated using the Tukey-Kramer post-hoc test, and these data are presented as mean \pm standard deviation. The rates of oocyte retrieval, 
oocyte maturity, fertilization, embryo cleavage, $\geq 7$ cells at day 3 , and blastocyst development were evaluated using the chi-square test and residual analysis. A $p$-value of $<0.05$ was considered to indicate statistical significance.

\section{Results}

Follicular diameter was correlated with the FF volume aspirated from individual follicles (Spearman rank-order correlation coefficient,
0.832; $p<0.001$, data not shown). The rate of oocyte retrieval was significantly lower in group A than in the other groups (46.0\% [86/ 187] vs. $67.5 \%-74.3 \%$ [172/255 to $124 / 167]$, respectively; $p<0.01$ ). The rate of MII oocyte retrieval was not significantly different among all groups (68.6\%-83.9\% [59/86 to 104/124]. In contrast, the rate of immature oocyte retrieval was significantly higher in group $A$ than in the other groups ( $25.6 \%$ [22/86] vs. $4.2 \%-14.3 \%$ [4/96 to $8 / 56$ ], respectively; $p<0.01)$. The rate of oocyte degeneration was not significantly different among all groups (Table 1).

Table 1. Relationship between the volume of follicular fluid aspirated from individual follicles and the rates of oocyte retrieval and maturity

\begin{tabular}{|c|c|c|c|c|c|c|c|}
\hline \multirow[b]{2}{*}{ Variable } & \multicolumn{6}{|c|}{ Volume of follicular fluid aspirated from individual follicles ( $\mathrm{mL})$} & \multirow[b]{2}{*}{$p$-value } \\
\hline & $\begin{array}{c}\text { Group A } \\
(<1.0)\end{array}$ & $\begin{array}{c}\text { Group B } \\
(1.0-<2.0)\end{array}$ & $\begin{array}{c}\text { Group C } \\
(2.0-<3.0)\end{array}$ & $\begin{array}{c}\text { Group D } \\
(3.0-<4.0)\end{array}$ & $\begin{array}{c}\text { Group E } \\
(4.0-<5.0)\end{array}$ & $\begin{array}{l}\text { Group F } \\
(\geq 5.0)\end{array}$ & \\
\hline No. of aspirated follicles & 187 & 255 & 167 & 141 & 84 & 80 & - \\
\hline No. of oocytes retrieved & $86(46.0)^{a)}$ & $172(67.5)$ & $124(74.3)^{\mathrm{a})}$ & $96(68.1)$ & $60(71.4)$ & $56(70.0)$ & $<0.001$ \\
\hline Mll oocytes & $59(68.6)$ & $139(80.8)$ & $104(83.9)$ & $78(81.3)$ & $46(76.7)$ & $42(75.0)$ & 0.123 \\
\hline Immature oocytes & $22(25.6)^{a)}$ & $19(11.0)$ & $14(11.3)$ & $4(4.2)^{a)}$ & $8(13.3)$ & $8(14.3)$ & 0.001 \\
\hline Degenerated oocytes & $5(5.8)$ & $14(8.1)$ & $6(4.8)$ & $14(14.6)$ & $6(10.0)$ & $6(10.7)$ & 0.157 \\
\hline
\end{tabular}

Values are presented as number (\%). The number of oocytes retrieved, MII oocytes, immature oocytes (metaphase I and germinal vesicle), and degenerated oocytes were evaluated using the chi-square test and residual analysis. A $p$-value of $<0.05$ was considered to indicate statistical significance.

MIl, metaphase II.

${ }^{a} p<0.01$

Table 2. Relationship between the volume of follicular fluid aspirated from individual follicles and oolemma stretchability during ICSI

\begin{tabular}{lccccccc}
\hline & \multicolumn{7}{c}{ Volume of follicular fluid aspirated from individual follicles (mL) } \\
\cline { 2 - 6 } Variable & $\begin{array}{c}\text { Group A } \\
(<1.0)\end{array}$ & $\begin{array}{c}\text { Group B } \\
(1.0-<2.0)\end{array}$ & $\begin{array}{c}\text { Group C } \\
(2.0-<3.0)\end{array}$ & $\begin{array}{c}\text { Group D } \\
(3.0-<4.0)\end{array}$ & $\begin{array}{c}\text { Group E } \\
(4.0-<5.0)\end{array}$ & $\begin{array}{c}\text { Group F } \\
(\geq 5.0)\end{array}$ & $p$-value \\
No. of Mll oocytes & 59 & 139 & 104 & 78 & 46 & 42 & - \\
Patient age (yr) & $38.6 \pm 4.7$ & $38.4 \pm 4.7$ & $38.6 \pm 4.6$ & $38.2 \pm 4.7$ & $38.0 \pm 5.0$ & $38.4 \pm 4.6$ & 0.974 \\
High oolemma stretchability & $46(78.0)^{\mathrm{a})}$ & $126(90.6)$ & $98(94.2)$ & $72(92.3)$ & $43(93.5)$ & $39(92.9)$ & 0.018 \\
Low oolemma stretchability & $13(22.0)^{\mathrm{a})}$ & $13(9.4)$ & $6(5.8)$ & $6(7.7)$ & $3(6.5)$ & $3(7.1)$ & \\
\hline
\end{tabular}

Values are presented as mean \pm standard deviation or number (\%). The mean age of patients was evaluated using one-way analysis of variance. Consequently, the mean age of patients was evaluated using the Tukey-Kramer post-hoc test. The rates of ovarian stimulation and stretchability were evaluated using the chisquare test and residual analysis. A $p$-value of $<0.05$ was considered to indicate statistical significance.

ICSI, intracytoplasmic sperm injection; MII, metaphase II.

a) $p<0.05$.

Table 3. Relationship between the volume of follicular fluid aspirated from individual follicles and the fertilization rate

\begin{tabular}{lccccccc}
\hline & \multicolumn{7}{c}{ Volume of follicular fluid aspirated from individual follicles $(\mathrm{mL})$} \\
\cline { 2 - 6 } Variable & $\begin{array}{c}\text { Group A } \\
(<1.0)\end{array}$ & $\begin{array}{c}\text { Group B } \\
(1.0-<2.0)\end{array}$ & $\begin{array}{c}\text { Group C } \\
(2.0-<3.0)\end{array}$ & $\begin{array}{c}\text { Group D } \\
(3.0-<4.0)\end{array}$ & $\begin{array}{c}\text { Group E } \\
(4.0-<5.0)\end{array}$ & $\begin{array}{c}\text { Group F } \\
(\geq 5.0)\end{array}$ & $p$-value \\
No. of MII oocytes & 59 & 139 & 104 & 78 & 46 & 42 & - \\
No. of 2PN2PB & $43(72.9)$ & $99(71.2)$ & $80(76.9)$ & $53(67.9)$ & $33(71.7)$ & $32(76.2)$ & 0.813 \\
No. of 1PN & $4(6.8)$ & $8(5.8)$ & $5(4.8)$ & $6(7.7)$ & $3(6.5)$ & $2(4.8)$ & 0.972 \\
No. of multiple PN & $2(3.4)$ & $2(1.4)$ & $2(1.9)$ & 0 & 0 & $1(2.4)$ & 0.597 \\
No. of unfertilized oocytes & $5(8.5)$ & $20(14.4)$ & $15(14.4)$ & $13(16.7)$ & $9(19.6)$ & $7(16.7)$ & 0.689 \\
No. of degenerated oocytes & $5(8.5)$ & $10(7.2)$ & $2(1.9)$ & $6(7.7)$ & $1(2.2)$ & 0 & 0.112 \\
\hline
\end{tabular}

Values are presented as number (\%). The rates of 2PNs2PBs oocytes, 1PN oocytes, multiple PN oocytes, unfertilized oocytes, and degenerated oocytes were evaluated using the chi-square test and residual analysis. A $p$-value of $<0.05$ was considered to indicate statistical significance.

MII, metaphase II; PN, pronucleus; PB, polar body. 
Table 4. Relationship between the volume of follicular fluid aspirated from individual follicles and embryo development

\begin{tabular}{lccccccc}
\hline & \multicolumn{7}{c}{ Volume of follicular fluid aspirated from individual follicles $(\mathrm{mL})$} \\
\cline { 2 - 6 } Variable & $\begin{array}{c}\text { Group A } \\
(<1.0)\end{array}$ & $\begin{array}{c}\text { Group B } \\
(1.0-<2.0)\end{array}$ & $\begin{array}{c}\text { Group C } \\
(2.0-<3.0)\end{array}$ & $\begin{array}{c}\text { Group D } \\
(3.0-<4.0)\end{array}$ & $\begin{array}{c}\text { Group E } \\
(4.0-<5.0)\end{array}$ & $\begin{array}{c}\text { Group F } \\
(\geq 5.0)\end{array}$ & $p$-value \\
\hline No. of 2PNs2PBs & 43 & 99 & 80 & 53 & 33 & 32 & - \\
Cleavage & $42 / 43(97.7)$ & $96 / 99(97.0)$ & $78 / 80(97.5)$ & $53 / 53(100.0)$ & $31 / 33(93.9)$ & $32 / 32(100.0)$ & 0.517 \\
$\geq 7$ cells at day 3 & $24 / 42(57.1)$ & $61 / 96(63.5)$ & $55 / 78(70.5)$ & $29 / 53(54.7)$ & $21 / 31(67.7)$ & $20 / 32(62.5)$ & 0.495 \\
No. of cultured oocytes until day 6 & 29 & 74 & 61 & 37 & 23 & 24 & - \\
Blastocysts & $13 / 29(44.8)$ & $44 / 74(59.5)$ & $39 / 61(63.9)$ & $19 / 37(51.4)$ & $14 / 23(60.9)$ & $9 / 24(37.5)$ & 0.203 \\
\hline
\end{tabular}

Values are presented as number (\%). The rates of cleavage, $\geq 7$ cells at day 3 and blastocyst development were evaluated using the chi-square test and residual analysis. A $p$-value of $<0.05$ was considered to indicate statistical significance.

$\mathrm{PN}$, pronucleus; $\mathrm{PB}$, polar body.

Data regarding the relationship between oolemma stretchability and FF volume during ICSI were also analyzed. The mean age of patients was not significantly different among all groups (Table 2). Oolemma stretchability was significantly less common in group $A$ than in the other groups during ICSI (22.0\% [13/59] vs. 5.8\%-9.4\% [6/104 to 13/139]; $p=0.018$ ) (Table 2).

There were no significant differences in the rates of oocytes with two pronuclei (PNs) and two polar bodies, oocytes with one PN, oocytes with multiple PNs, unfertilized oocytes, and degenerated oocytes after ICSI among all groups (Table 3). Moreover, regarding embryo development, there were no significant differences in the rates of embryo cleavage, $\geq 7$ cells at day 3 , and blastocyst development after ICSI among all groups (Table 4). A relationship was noted between FF volume and MII oolemma stretchability.

\section{Discussion}

This study demonstrated that the oolemma of MII oocytes from individual follicles with a low FF volume were more likely to have low stretchability. This result suggests that the FF volume of individual follicles may be associated with MII oolemma stretchability.

The present data show that the FF volume was correlated with follicular diameter, which is consistent with the findings of Arashiro et al. [22]. We focused on the relationship between follicle shape and follicle diameter under two-dimensional transvaginal ultrasonography. Round and polygonal follicles tended to have the largest mean diameter. However, follicles are rarely spherical; most are elliptical, owing to follicular overcrowding. Thus, the volume of ellipsoid follicles measured with ultrasonography was overestimated as often as it was underestimated $[23,24]$. Accordingly, we investigated the relationship between FF volume and oolemma stretchability.

The present results indicate that the rate of oocyte retrieval from $<1.0 \mathrm{~mL}$ of FF was low, and even if the $\mathrm{COC}$ was obtained, the rate of immature oocyte retrieval was high. Lee et al. [25] showed that the rate of mature oocyte retrieval was low when the FF volume was
$<3.0 \mathrm{~mL}$ and that the FF volume was related to oocyte maturity. Moreover, previous studies have reported that a low rate of oocyte maturity was associated with a small follicular diameter $[17,26]$. Accordingly, the present results show that FF volume and follicular diameter were related to the rate of oocyte maturity.

In this study, low oolemma stretchability was significantly more common when the aspirated FF volume was $<1.0 \mathrm{~mL}$ during ICSI. A previous study showed that the follicular size was related to the contents of the FF, such as the levels of steroid hormones [14], which can affect the oocyte quality of individual follicles $[15,16]$. Therefore, we speculate that differences in the microenvironment, such as the steroid hormone levels in the $\mathrm{FF}$, are potentially associated with oolemma stretchability during ICSI.

In a previous study with mice, a significantly less viscous cytoplasm was noted for younger eggs (8-12 weeks old) than for older eggs (40-45 weeks old, near the end of the reproductive lifespan; an analog to human female infertility associated with advanced maternal age [ $\geq 35$ years]) [27]. These results suggest that oocyte quality, represented by factors such as the cytoplasm and oolemma, may be lower for patients of advanced maternal age. In this study, the mean age of the patients was not different among all the groups, suggesting that human oolemma stretchability was not related to the age of the patients. Hence, the main cause of low stretchability in this study is unlikely to have been the age of the patients.

Several studies have reported on the relationship between follicular size/volume and the fertilization rate following ICSI [17,25]. In one study, the fertilization rate following ICSI was affected by follicular size [17]. In contrast, Lee et al. [25] reported that FF volume did not affect the fertilization rate, as FF volumes of $<3.0 \mathrm{~mL}, 3.0$ to $\leq 5.0$ $\mathrm{mL}$, and $>5.0 \mathrm{~mL}$ were associated with fertilization rates of $72.15 \%$, $77.99 \%$, and $72.47 \%$, respectively. The present study showed that FF volume was not associated with the rates of fertilization and oocyte degeneration.

Moreover, we found that FF volume was not associated with the rates of embryo cleavage, $\geq 7$ cells at day 3 , and blastocyst develop- 
ment. This is consistent with Salha et al. [19], who showed that follicular size and oocyte development factors, such as cleavage, implantation, clinical pregnancy, and live birth, were not closely related and were independent of each other.

The present study has some limitations because of the retrospective nature of the analysis. First, the relationships between FF volume and the rates of chemical/clinical pregnancy and live birth were not analyzed because we frequently performed two-step embryo transfer [28] or implanted two embryos/blastocysts into a patient's uterus according to the guidelines of the Japan Society for Obstetrics and Gynecology [29]. Second, because the levels of the FF contents, such as steroid hormones, transforming growth factor- $\beta$, and interleukins, in independent follicles were not measured in this study, we cannot describe the characteristics of FF composition in detail. Third, the oolemma stretchability analysis may have been less objective than force measurement using an oocyte microinjection system $[27,30]$. However, to ensure objectivity, the category of high stretchability was included regardless of the distance between the penetration point of the oolemma and the edge of the injection pipette during aspiration.

Moreover, cytoplasmic viscosity [4] and morphology [31] of the oocyte are not related to the stimulation protocol. In contrast, the quality of the zona pellucida is dependent on the ovarian stimulation protocol [32]. Future studies investigating the effects of the GnRH agonist protocol on MII oolemma stretchability are planned.

In conclusion, FF volume is potentially associated with human oolemma stretchability during ICSI. To date, during ovarian stimulation, coordination of follicular size and oocyte maturity has been the focus. For the coordination of follicular development in ovarian stimulation, pretreatment with luteal estradiol [33] or oral contraceptive pills [34] is performed, and a decrease in follicle-stimulating hormone is prevented in the late follicular phase [35]. In terms of oolemma stretchability and oocyte maturity, ensuring uniform follicular size during ovarian stimulation is crucial for obtaining good-quality oocytes.

\section{Conflict of interest}

No potential conflict of interest relevant to this article was reported.

\section{References}

1. Mansour R, Fahmy I, Tawab NA, Kamal A, El-Demery Y, Aboulghar $M$, et al. Electrical activation of oocytes after intracytoplasmic sperm injection: a controlled randomized study. Fertil Steril 2009;91:133-9.

2. Richter KS, Davis A, Carter J, Greenhouse SJ, Mottla GL, Tucker MJ.
No advantage of laser-assisted over conventional intracytoplasmic sperm injection: a randomized controlled trial [NCT001 14725]. J Exp Clin Assist Reprod 2006;3:5.

3. Krause I, Pohler U, Grosse S, Shebl O, Petek E, Chandra A, et al. Characterization of the injection funnel during intracytoplasmic sperm injection reflects cytoplasmic maturity of the oocyte. Fertil Steril 2016;106:1101-6.

4. Ebner $T$, Moser $M$, Sommergruber $M$, Puchner $M$, Wiesinger $R$, Tews $\mathrm{G}$. Developmental competence of oocytes showing increased cytoplasmic viscosity. Hum Reprod 2003;18:1294-8.

5. Iwayama H, Hochi S, Yamashita M. Low stretching ability of human oolemma during piezo-ICSI as a risk factor on post-injection survival and implantation. J Mamm Ova Res 2010;27:150-6.

6. Yanagida K, Katayose H, Suzuki K, Suganuma A, Sato A. Flexibility of oolemma is an important factor for oocyte survival after ICSI. J Mamm Ova Res 2001;18:93-8.

7. Mizobe Y, Oya N, Iwakiri R, Yoshida N, Sato Y, Onoue N, et al. Developmental ability of embryos produced from oocytes with fragile oolemma by intracytoplasmic sperm injection. J Assist Reprod Genet 2016;33:1685-90.

8. Commentary: oocyte degeneration after ICSI. J Reprod Stem Cell Biotechnol 2010;1:193-211.

9. Yavas Y, Roberge S, Khamsi F, Shirazi P, Endman MW, Wong JC. Performing ICSI using an injection pipette with the smallest possible inner diameter and a long taper increases normal fertilization rate, decreases incidence of degeneration and tripronuclear zygotes, and enhances embryo development. J Assist Reprod Genet 2001;18:426-35.

10. Hiraoka K, Kitamura S. Clinical efficiency of Piezo-ICSI using micropipettes with a wall thickness of $0.625 \mu \mathrm{m}$. J Assist Reprod Genet 2015;32:1827-33.

11. Rosen MP, Shen S, Dobson AT, Fujimoto VY, McCulloch CE, Cedars Ml. Oocyte degeneration after intracytoplasmic sperm injection: a multivariate analysis to assess its importance as a laboratory or clinical marker. Fertil Steril 2006;85:1736-43.

12. Revelli A, Delle Piane L, Casano S, Molinari E, Massobrio M, Rinaudo P. Follicular fluid content and oocyte quality: from single biochemical markers to metabolomics. Reprod Biol Endocrinol 2009;7:40

13. Dumesic DA, Meldrum DR, Katz-Jaffe MG, Krisher RL, Schoolcraft WB. Oocyte environment: follicular fluid and cumulus cells are critical for oocyte health. Fertil Steril 2015;103:303-16.

14. van Dessel HJ, Schipper I, Pache TD, van Geldorp H, de Jong FH, Fauser BC. Normal human follicle development: an evaluation of correlations with oestradiol, androstenedione and progesterone levels in individual follicles. Clin Endocrinol (Oxf) 1996;44:191-8.

15. Mendoza C, Cremades N, Ruiz-Requena E, Martinez F, Ortega E, 
Bernabeu S, et al. Relationship between fertilization results after intracytoplasmic sperm injection, and intrafollicular steroid, pituitary hormone and cytokine concentrations. Hum Reprod 1999; 14:628-35.

16. Lamb JD, Zamah AM, Shen S, McCulloch C, Cedars MI, Rosen MP. Follicular fluid steroid hormone levels are associated with fertilization outcome after intracytoplasmic sperm injection. Fertil Steril 2010;94:952-7.

17. Rosen MP, Shen S, Dobson AT, Rinaudo PF, McCulloch CE, Cedars MI. A quantitative assessment of follicle size on oocyte developmental competence. Fertil Steril 2008;90:684-90.

18. Rodriguez-Fuentes A, Hernandez J, Garcia-Guzman R, Chinea E, laconianni L, Palumbo A. Prospective evaluation of automated follicle monitoring in 58 in vitro fertilization cycles: follicular volume as a new indicator of oocyte maturity. Fertil Steril 2010;93:616-20.

19. Salha O, Nugent D, Dada T, Kaufmann S, Levett S, Jenner L, et al. The relationship between follicular fluid aspirate volume and oocyte maturity in in-vitro fertilization cycles. Hum Reprod 1998; 13:1901-6.

20. Inoue T, Ono $Y$, Emi N. Preservation of spermatozoa by refrigeration. In: Frias TA, Cano MJ, editors. Pregnancy: risk factors, management and recovery. New York: Nova Science Publishers; 2012. p. 121-46.

21. Inoue T, Sugimoto H, Okubo K, Emi N, Matsushita Y, Kojima K, et al. Successful pregnancy after intracytoplasmic sperm injection with testicular spermatozoa transported only under refrigeration. Reprod Med Biol 2010;9:173-7.

22. Arashiro EK, Palhao MP, Wohlres-Viana S, Siqueira LG, Camargo $L S$, Henry $M$, et al. In vivo collection of follicular fluid and granulosa cells from individual follicles of different diameters in cattle by an adapted ovum pick-up system. Reprod Biol Endocrinol 2013;11:73.

23. Raine-Fenning $\mathrm{N}$, Jayaprakasan $\mathrm{K}$, Clewes J, Joergner I, Bonaki SD, Chamberlain S, et al. SonoAVC: a novel method of automatic volume calculation. Ultrasound Obstet Gynecol 2008;31:691-6.

24. Penzias AS, Emmi AM, Dubey AK, Layman LC, DeCherney AH, Reindollar RH. Ultrasound prediction of follicle volume: is the mean diameter reflective? Fertil Steril 1994;62:1274-6.

25. Lee TF, Lee RK, Hwu YM, Chih YF, Tsai YC, Su JT. Relationship of follicular size to the development of intracytoplasmic sperm in- jection-derived human embryos. Taiwan J Obstet Gynecol 2010; 49:302-5.

26. Mehri S, Levi Setti PE, Greco K, Sakkas D, Martinez G, Patrizio P. Correlation between follicular diameters and flushing versus no flushing on oocyte maturity, fertilization rate and embryo quality. J Assist Reprod Genet 2014;31:73-7.

27. Liu X, Fernandes R, Jurisicova A, Casper RF, Sun Y. In situ mechanical characterization of mouse oocytes using a cell holding device. Lab Chip 2010;10:2154-61.

28. Yazbeck C, Ben Jamaa N, Hazout A, Cohen-Bacrie P, Junca AM, Rougier N. Advantages of the two-step embryo transfer strategy in human IVF/ICSI cycles. Zygote 2013;21:77-83.

29. Japan Society of Obstetrics and Gynecology. Prevention of multiple pregnancy in assisted reproductive technology. Acta Obstet Gynaecol Jpn 2014;8:1918.

30. Liu X, Shi J, Zong Z, Wan KT, Sun Y. Elastic and viscoelastic characterization of mouse oocytes using micropipette indentation. Ann Biomed Eng 2012;40:2122-30.

31. Cota AM, Oliveira JB, Petersen CG, Mauri AL, Massaro FC, Silva LF, et al. GnRH agonist versus $\mathrm{GnRH}$ antagonist in assisted reproduction cycles: oocyte morphology. Reprod Biol Endocrinol 2012; 10:33.

32. Ebner T, Balaban B, Moser M, Shebl O, Urman B, Ata B, et al. Automatic user-independent zona pellucida imaging at the oocyte stage allows for the prediction of preimplantation development. Fertil Steril 2010;94:913-20.

33. Fanchin R, Schonauer LM, Cunha-Filho JS, Mendez Lozano DH, Frydman R. Coordination of antral follicle growth: basis for innovative concepts of controlled ovarian hyperstimulation. Semin Reprod Med 2005;23:354-62.

34. Damario MA, Barmat L, Liu HC, Davis OK, Rosenwaks Z. Dual suppression with oral contraceptives and gonadotrophin releasing-hormone agonists improves in-vitro fertilization outcome in high responder patients. Hum Reprod 1997;12:2359-65.

35. Schipper I, Hop WC, Fauser BC. The follicle-stimulating hormone (FSH) threshold/window concept examined by different interventions with exogenous FSH during the follicular phase of the normal menstrual cycle: duration, rather than magnitude, of FSH increase affects follicle development. J Clin Endocrinol Metab 1998;83:1292-8. 